\title{
Effect of Mineral-Oil Addition on Film Stability of Polar Lipid Constituents Derived from Meibum
}

\author{
Masataka Yoshida ${ }^{1}$, Masato Yamaguchi ${ }^{2}$, Atsushi Sato $^{3}$, Miyuki Miyake ${ }^{3}$, \\ Nobuhito Tabuchi ${ }^{1}$, Ryo Kon ${ }^{1}$, and Ken-ichi limura ${ }^{4 *}$ \\ ${ }^{1}$ Pharmaceutical Research Laboratories, Research and Development Headquarters, Lion Corporation, 100 Tajima, Odawara, Kanagawa \\ 256-0811, JAPAN \\ ${ }^{2}$ Department of Material and Environmental Chemistry, Graduate School of Engineering, Utsunomiya University, 7-1-2 Yoto, Utsunomiya, Tochigi \\ 321-8585, JAPAN \\ ${ }^{3}$ Advanced Analytical Science Laboratories, Research and Development Headquarters, Lion Corporation, 7-2-1 Hirai, Edogawa-ku, Tokyo \\ 132-0035, JAPAN \\ ${ }^{4}$ Division of Engineering and Agriculture, Graduate School of Regional Development and Creativity, Utsunomiya University, 7-1-2 Yoto, \\ Utsunomiya, Tochigi 321-8585, JAPAN
}

\begin{abstract}
We studied the effects of mineral oil (MO) on the properties and structure of a spread monolayer of polar lipid constituents in meibum, by performing cyclic lateral compression-expansion experiments using a Langmuir trough. A meibum sample without nonpolar lipids (meibum $\Delta$ nonpolar-lipid) was prepared by removing the nonpolar lipids from biological meibum extruded from rabbit eyelids and spread on a water surface for measuring the cyclic surface pressure $(\pi)$-film area $(A)$ isotherms with in situ observation of the film morphology using a Brewster angle microscope. The meibum $\Delta$ nonpolar-lipid formed a homogeneous fluid monolayer and underwent collapse upon compression. The $\pi-A$ isotherm shifted to a smaller area upon repeating the compression-expansion cycles. These observations contrasted those obtained for meibum previously, which may have resulted from the absence of nonpolar lipids. The recovery of the film stability against the lateral compression-expansion cycles was analyzed by adding MO as a nonpolar compound to the film system. A spread film of 1:1 mixture (by weight) could recover the high reversibility of the $\pi-A$ isotherms during the repeated compression and expansion processes.
\end{abstract}

Key words: meibum, mineral oil, spread monolayer, surface pressure-film area isotherm, Brewster angle microscopy

\section{Introduction}

The tear film on the eye is composed of three layers lipid, aqueous, and mucin layers, from the surface to the inside $^{1}$. The lipid layer (called tear film lipid layer: TFLL) is formed by a lipid mixture (meibum) secreted from the meibomian gland ${ }^{2}$, and it plays an important role in maintaining eye health. It has been reported that meibum includes compounds such as wax esters $(\sim 43 \%)$, cholesteryl esters ( $\sim 49 \%)$, phospholipids $(<1 \%)$, sphingolipids ( $\sim 2 \%)$, triglycerides $(\sim 2 \%)$, cholesterol $(\sim 2 \%)$, and (O-acyl) $\omega$-hydroxy fatty acids $(\sim 3 \%)^{3)}$. These lipids form two distinguishable layers in the TFLL - a nonpolar lipid layer primarily comprised of wax esters and cholesteryl esters and a polar lipid layer of phospholipids, free fatty acids, and so on ${ }^{4)}$. Many studies have been conducted to elucidate the film structures and functions of TFLL. It has been proposed that lipid microcrystals dispersed in the nonpolar lipid layer provide elasticity to the TFLL ${ }^{5)}$. The TFLL is viscoelastic and can exist stably on the surface of water for a long time to prevent the evaporation of water from the tear film $^{6)}$. Furthermore, the TFLL can maintain its stable structure against the mechanical stimuli of compression and expansion, which occur repeatedly upon blinking ${ }^{7}$.

Spread monolayers are two-dimensional systems useful for studying the physiochemical properties, intermolecular interactions, and self-organization phenomena of water-insoluble molecules confined at the air/water interface ${ }^{8,9)}$. Although studies have been motivated by a variety of scientific interests, the use of monolayers as models of biological interfaces has attracted significant attention for understanding biological events and developing functional thin films ${ }^{9-12)}$. An advantage of the Langmuir balance technique is that the lateral compression of the monolayer by movable barrier $(\mathrm{s})$ enables control of the molecular area

\footnotetext{
*Correspondence to: Ken-ichi limura, Division of Engineering and Agriculture, Graduate School of Regional Development and Creativity, Utsunomiya University, 7-1-2 Yoto, Utsunomiya, Tochigi 321-8585, JAPAN

E-mail: emlak@cc.utsunomiya-u.ac.jp

Accepted February 4, 2020 (received for review January 6, 2020)

Journal of Oleo Science ISSN 1345-8957 print / ISSN 1347-3352 online

http://www.jstage.jst.go.jp/browse/jos/ http://mc.manusriptcentral.com/jjocs
} 
and state and helps analyze the dynamic behavior of monolayers on the water surface ${ }^{9)}$. On the eye surface, the TFLL on the aqueous layer is compressed and expanded repeatedly upon blinking, which can be mimicked by using a spread monolayer of meibum on the water surface. To explore the mechanism of TFLL stability against the mechanical stimulus caused by blinking, structural changes resulting from the compression-expansion processes have been investigated for Langmuir films of meibum and their analogues prepared at the air/water interface ${ }^{7,13-17)}$. We have also evaluated the properties and structures of spread films of biological meibum obtained from rabbit eyelids, phospholipid-removed meibum, and phospholipid/cholesterol-removed meibum through cyclic compression-expansion experiments using the Langmuir film balance technique and observations made using a Brewster angle microscope $(\mathrm{BAM})^{18)}$. The spread film of meibum is composed of condensed-phase networks, primarily consisting of nonpolar lipids and a monolayer phase of polar lipids between the networks. Although the nonpolar lipid networks are gathered by compression, their direct contact and subsequent coalescence seem to have been prevented by the formation of collapsed aggregates of polar lipids confined by the networks. Both the isotherms and morphological changes in the compression-expansion cycles are highly reversible, which can be attributed to the deformability and resiliency of nonpolar lipid networks and the reversible collapse and spreading properties of the polar lipid monolayer phase.

Generally, insoluble monolayers of amphiphilic materials such as phospholipids are collapsed irreversibly by compression $^{19,20)}$. However, as mentioned above, polar lipid monolayers surrounded by nonpolar lipid networks in the spread film of meibum return to their original states after expansion following compression ${ }^{18)}$. On the other hand, as described in this paper, a spread monolayer of polar lipids derived from meibum exhibited a shift of the surface pressure $(\pi)$-film area $(A)$ isotherm, to a smaller area, following successive compression-expansion cycles. This shift may originate from the lack of nonpolar components in the monolayer system, and thus, in the present study, we focus on the effects of nonpolar components on the stability of a polar lipid monolayer subjected to repeated compression-expansion cycles. An overview of the experimental system and meibum samples used in this research are presented schematically in Fig. 1. The polar lipid sample was prepared by removing nonpolar lipids such as wax esters and cholesteryl esters from biological meibum. This sample was named meibum $\Delta$ nonpolar-lipid and used in the cyclic compression-expansion experiments. It was ob- (a) Experimental system

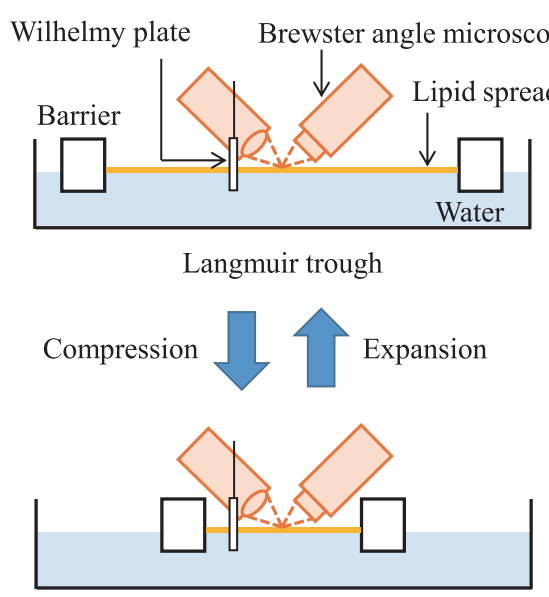

(b) Meibum film

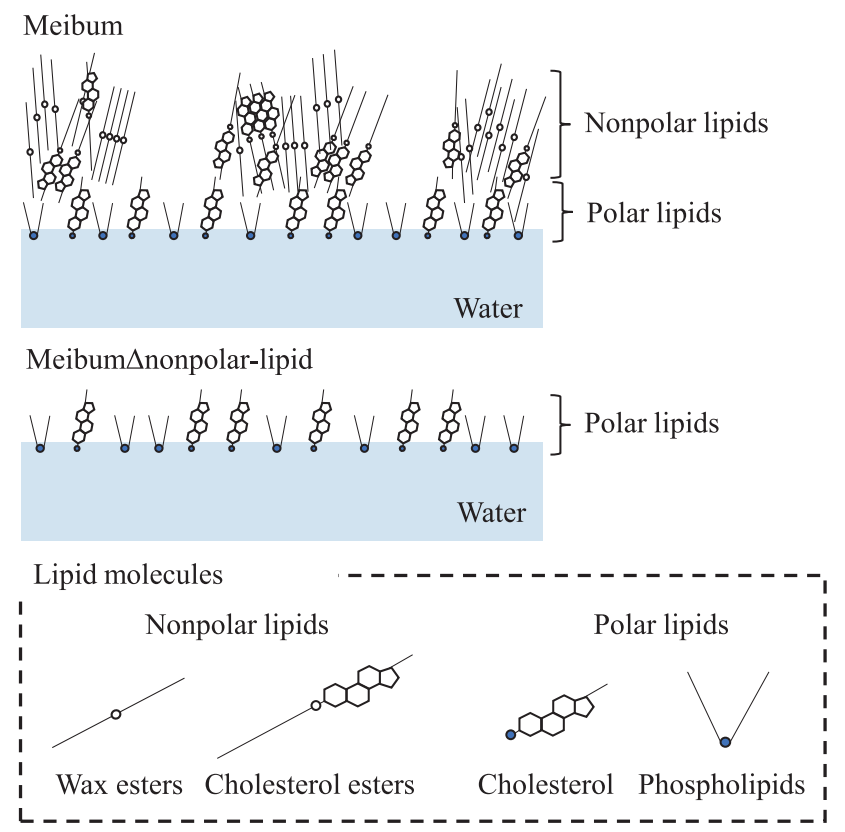

Fig. 1 A schematic overview of experimental system and meibum samples used in this research. (a) In a Langmuir trough, ultrapure water was filled, and a lipid film was spread on it. Surface pressure was measured by a Wilhelmy-type surface balance as a function of film area. Film morphology is observed by Brewster angle microscope. (b) (Top) The meibum film has been thought to be composed of a monolayer of polar lipids such as cholesterol and phospholipids attached to the water surface and a layer of non-polar lipids such as wax esters and cholesterol esters on the polar lipid layer. (Middle) In this study, nonpolar lipids were removed from biological meibum, and the resultant sample,

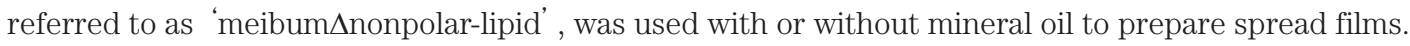


served that the shift in the cyclic $\pi-A$ isotherms for the meibum $\Delta$ nonpolar-lipid spread monolayer was suppressed by adding mineral oil (MO) as a nonpolar component.

\section{Experimental Procedures}

\subsection{Preparation of meibum $\Delta$ nonpolar-lipid}

The details of the procedures used for preparing the meibum samples have been described in a previous work ${ }^{18)}$. Briefly, meibum was obtained from rabbit eyelids. A chloroform solution of the obtained meibum was added to a silica gel column that was eluted, first with chloroform and then with methanol. The eluted fractions were analyzed using thin-layer chromatography, and the fractions without nonpolar lipids were combined to obtain the meibum$\Delta$ nonpolar-lipid sample.

\subsection{Lipid analysis}

Lipid analysis was performed to examine the components of the meibum and meibum $\Delta$ nonpolar-lipid samples. First, chloroform solutions of meibum and meibum$\Delta$ nonpolar-lipid were dried through an $\mathrm{N}_{2}$ gas purge. The dried materials were dissolved in chloroform/methanol
(1/1) to prepare sample solutions for lipid analysis using a liquid chromatography (LC)-mass spectroscopy (MS) device composed of an LC instrument (UPLC I-Class; Waters Corporation) and a mass spectrometer (Q Exactive Plus; Thermo Fisher Scientific K.K.). Electrospray ionization was selected as the ionization method. The data were analyzed using manufacturer-provided software (TraceFinder; Thermo Fisher Scientific K.K.), and the peak areas were extracted automatically.

\subsection{Measurement of $\pi-A$ isotherms and observation of film morphology}

A chloroform solution of meibum $\Delta$ nonpolar-lipid or MO (HICALL M-352; Kaneda Co., Ltd.)-added meibum$\Delta$ nonpolar-lipid (hereafter called $\mathrm{MO} / \mathrm{meibum} \Delta$ nonpolarlipid) was spread on an ultrapure water surface at $20^{\circ} \mathrm{C}$ in a Langmuir trough (NIMA, Large) equipped with a BAM(EP3BAM, Biolin Scientific). The mixing ratios of MO:meibum$\Delta$ nonpolar-lipid were $0.2: 1,1: 1$, and $10: 1$ by weight. The first compression began 30 min after spreading. The compression-expansion cycles of the $\pi-A$ isotherm measurement were applied at a rate of $510 \mathrm{~cm}^{2} / \mathrm{min}$ and repeated five times. BAM images were taken before compression and expansion in the cycles.

(a) Polar lipids
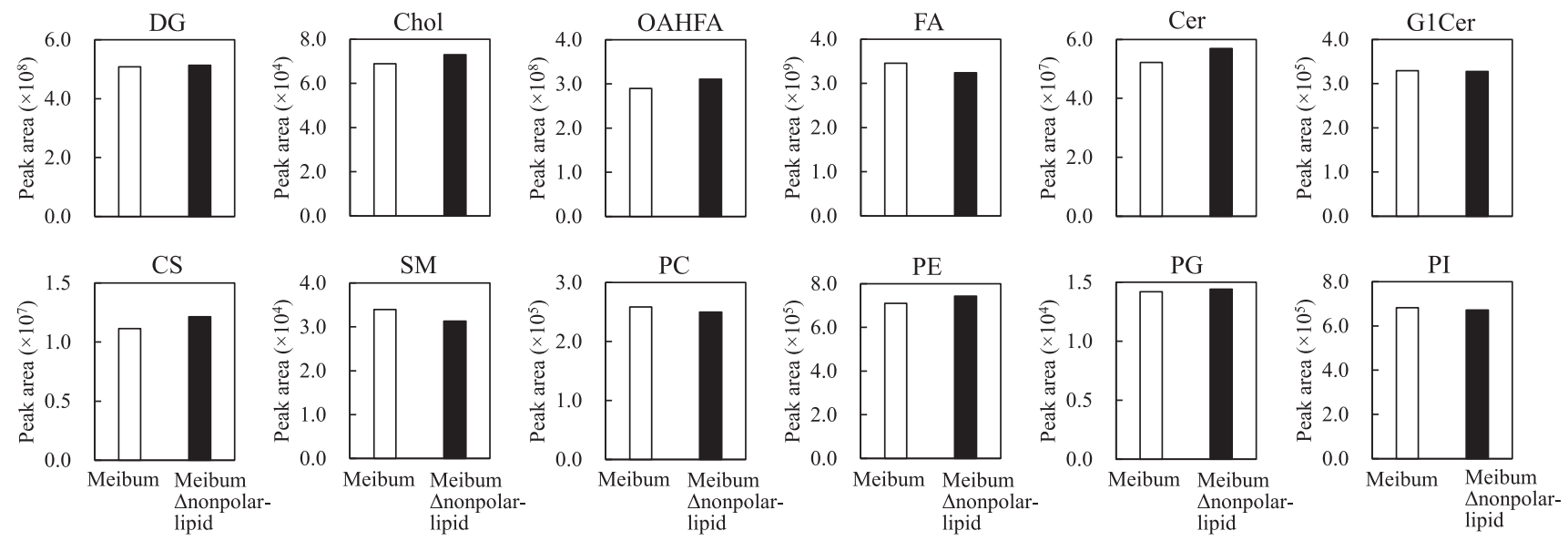

(b) Nonpolar lipids
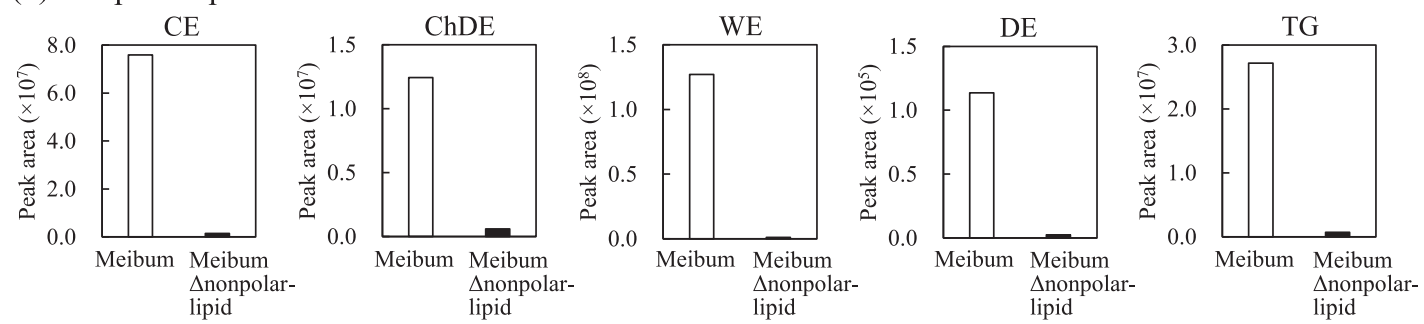

Fig. 2 MS peak areas for lipids in meibum and meibum $\Delta$ nonpolar-lipid. (a) Polar lipids: diglycerides (DG), cholesterol (Chol), O-acyl $\omega$-hydroxy fatty acids (OAHFA), free fatty acids (FA), ceramides (Cer), monoglycosyl ceramides (G1Cer), cholesterol sulfate (CS), sphingomyelin (SM), phosphatidylcholine (PC), phosphatidylethanolamine (PE), phosphatidylglycerol (PG), phosphatidylinositol(PI). (b) Nonpolar lipids: cholesterol esters (CE), $\omega$ type I -St diesters (ChDE), wax esters (WE), $\alpha, \omega$ type II diesters (DE), triglycerides (TG). 


\section{Results}

3.1 Lipid components in meibum and meibum $\Delta$ nonpolarlipid

The results of the lipid analysis of meibum and meibum$\Delta$ nonpolar-lipid are summarized in Fig. 2. The MS peak areas of polar lipids in meibum $\Delta$ nonpolar-lipid - namely, diglycerides (DG), cholesterol (Chol), O-acyl $\omega$-hydroxy fatty acids (OAHFA), free fatty acids (FA), ceramides (Cer), monoglycosyl ceramides (G1Cer), cholesterol sulfate (CS), sphingomyelin (SM), phosphatidyl choline (PC), phosphatidyl ethanolamine (PE), phosphatidyl glycerol (PG), and phosphatidyl inositol(PI) - were equivalent (within \pm $10 \%)$ to those in meibum. In comparison, the peak areas of nonpolar lipids in meibum $\Delta$ nonpolar-lipid - namely, cholesterol esters (CE), $\omega$ type I -St diesters (ChDE), wax esters (WE), $\alpha, \omega$ type II diesters (DE), and triglycerides (TG) were approximately less than one-tenth of those in meibum. These data clearly indicate that the meibum$\Delta$ nonpolar-lipid sample is composed solely of polar lipids.

\subsection{Meibum $\Delta$ nonpolar-lipid film}

Figure 3 shows the (a) cyclic $\pi-A$ isotherms and $(b-f)$ BAM images of a spread monolayer of meibum $\Delta$ nonpolarlipid. In the first compression, the surface pressure started increasing monotonously, at approximately $100 \times 10^{5} \mathrm{~cm}^{2} / \mathrm{g}$. The BAM observations suggested that the monolayer was in a homogeneous fluid phase before the first compression after spreading (image (b)), and no change was observed in the monolayer homogeneity during compression, up to a kink in the isotherm at approximately $15.6 \mathrm{mN} / \mathrm{m}$. Further compression above the kink to a smaller area induced the formation of aggregate formation (image (c)), most likely due to the monolayer collapse. In the first expansion process, the isotherm almost followed the compression process, and the aggregates disappeared (image(d)).
However, interestingly, the isotherm shifted to a smaller area as the cycles progressed, although BAM images repeatedly indicated the formation of a homogeneous monolayer in the expanded film and of aggregates in the fully compressed film during the second and fifth cycle (image (d) and (f)).

\subsection{MO-added meibum $\Delta$ nonpolar-lipid film}

As an attempt to improve the stability of the meibum$\Delta$ nonpolar-lipid monolayer on the water surface in the repeated compression-expansion cycles, MO was added to the film system, as a nonpolar substance. MO was mixed with meibum $\Delta$ nonpolar-lipid in chloroform and spread on the water surface. Figure 4 shows the $\pi-A$ isotherms and BAM images for a MO/meibum $\Delta$ nonpolar-lipid (0.2:1) film. The isotherms suggest that the film was expanded and that a kink appeared at the surface pressure of approximately $13.5 \mathrm{mN} / \mathrm{m}$. The compression and expansion isotherms in each cycle were in good agreement with each other, but the isotherms still shifted to lower area with the increase in cycle number. These features resemble those of the meibum $\Delta$ nonpolar-lipid monolayer. A BAM image observed before the first compression (image $(b)$ ) revealed the presence of some granular spots, which could be the MO or MO-rich phase separated from the surrounding monolayer. In the fully compressed film (image (c) and (e)), the surface density of bright spots seemed to have increased; however, some could be collapsed aggregates, as it is difficult to distinguish between the two. When the surface pressure was released to zero in the expansion process, the film morphology retuned to one similar to that of the uncompressed one (image (d) and (f)). Thus, the morphological change also exhibited reversibility during the compression and expansion processes. It is worth noting that the kink in the isotherm was observed at a surface pressure lower than (b) before $1 \mathrm{st}$ com. at $0 \mathrm{mN} / \mathrm{m}$

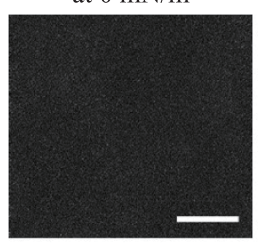

(c) before 1 st ex. at $17 \mathrm{mN} / \mathrm{m}$ (d) before 2 nd com. at $0 \mathrm{mN} / \mathrm{m}$

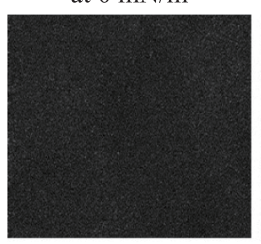

(e) before $2 \mathrm{nd}$ ex at $18 \mathrm{mN} / \mathrm{m}$
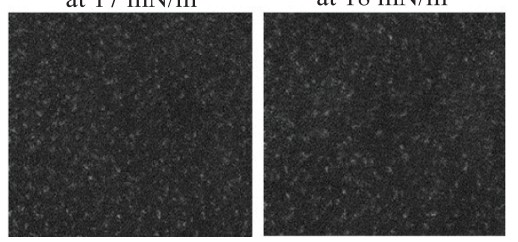

(f) after 5 th ex. at $0 \mathrm{mN} / \mathrm{m}$

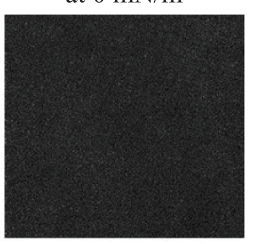

$+2$

Fig. 3 (a) $\pi-A$ isotherms and $(b-f)$ BAM images of meibum $\Delta$ nonpolar-lipid film in compression-expansion cycles (five times) at $20^{\circ} \mathrm{C}$. The images (b), (d), and (f) were taken at $0 \mathrm{mN} / \mathrm{m}\left(A \approx 146 \times 10^{5} \mathrm{~cm}^{2} / \mathrm{g}\right)$ just before starting the first and second compressions and after the fifth expansion, respectively. The images (c)and (e) were observed at 17 and $18 \mathrm{mN} / \mathrm{m}$ just before starting the first and second expansions, respectively. A bar in (b) corresponds to $50 \mu \mathrm{m}$. 
that for the monolayer of meibum $\Delta$ nonpolar-lipid, suggesting that the monolayer region did not consist of meibum$\Delta$ nonpolar-lipid molecules alone.

When MO was mixed with meibum $\Delta$ nonpolar-lipid in a $1: 1$ ratio by weight, the resultant spread film produced cyclic $\pi-A$ isotherms with a gentle kink at approximately $12.5 \mathrm{mN} / \mathrm{m}$ (Fig. $5(\mathrm{a})$ ). In addition to the compression and expansion isotherms in a single cycle, those in the first to fifth cycles almost coincided with each other, which was noticeably different from the case in the above-mentioned two films. In a BAM image after spreading(image(b)), granular spots like those in the film of $\mathrm{MO} / \mathrm{meibum}-$ $\Delta$ nonpolar-lipid $(0.2: 1)$ were observed. The spots were gathered upon compression, and could be coexistent with the aggregates above the surface pressure of the kink (image $(c))$. After the first cycle $(\operatorname{image}(d))$, the film ex- hibited many interconnected spots, presumably formed by the occasional contact of the spots in the compressed film followed by lateral flow in the surrounding fluid monolayer upon expansion. From the second to the fifth cycle, the interconnected spots were gathered and redispersed repeatedly upon compression and expansion, respectively.

The addition of MO to meibum $\Delta$ nonpolar-lipid at the mixing ratio of 10:1 largely decreased the onset point of surface pressure rise in the isotherms and increased the number density of spots in the BAM images, as expected (Fig. 6). One may think that there is hysteresis between the compression and expansion isotherms at relatively high surface pressures. However, considering the scale of the area axis, the hysteresis would be negligible, and the isotherms could be judged to be highly reversible. It is also interesting to note that any kink corresponding to those ob- (a) $\pi$ - $A$ isotherms

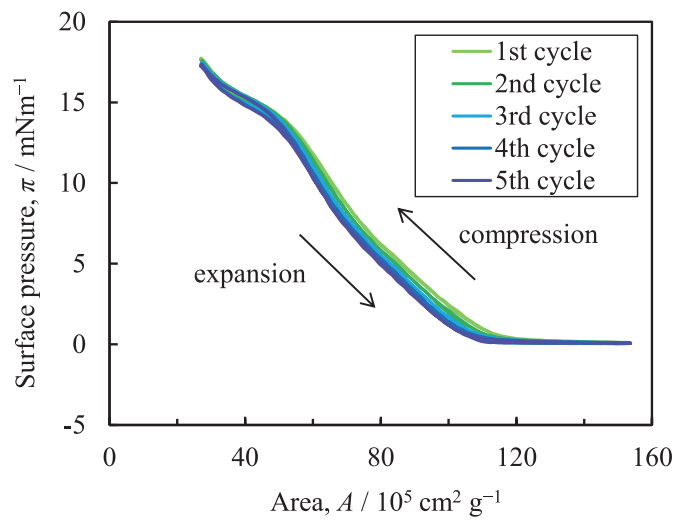

(b) before 1 st com. at $0 \mathrm{mN} / \mathrm{m}$

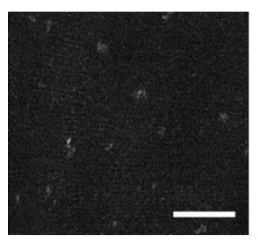

(c) before $1 \mathrm{st}$ ex at $18 \mathrm{mN} / \mathrm{m}$ (d) before 2 nd com. at $0 \mathrm{mN} / \mathrm{m}$

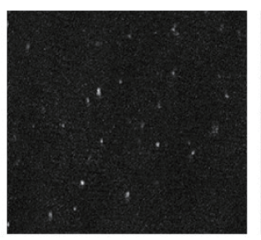

(e) before $2 \mathrm{nd} \mathrm{ex}$ at $18 \mathrm{mN} / \mathrm{m}$
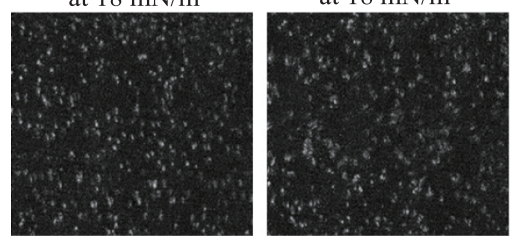

(f) after 5 th ex. at $0 \mathrm{mN} / \mathrm{m}$

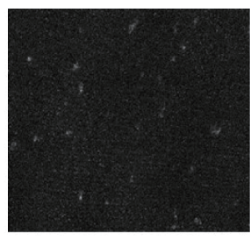

Fig. 4 (a) $\pi-A$ isotherms and (b-f) BAM images of MO/meibum $\Delta$ nonpolar-lipid (0.2:1) film in compression-expansion cycles (five times) at $20^{\circ} \mathrm{C}$. The images (b), (d), and (f) were taken at $0 \mathrm{mN} / \mathrm{m}\left(A \approx 151 \times 10^{5} \mathrm{~cm}^{2} / \mathrm{g}\right.$ ) just before starting the first and second compressions and after the fifth expansion, respectively. The images (c) and (e) were observed at 18 $\mathrm{mN} / \mathrm{m}$ just before starting the first and second expansions, respectively. A bar in (b) corresponds to $50 \mu \mathrm{m}$.

(a) $\pi$ - $A$ isotherms

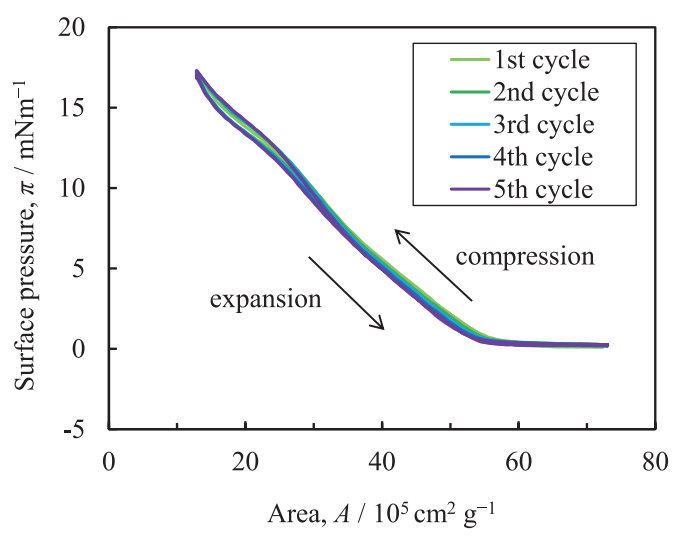

(b) before 1 st com. at $0 \mathrm{mN} / \mathrm{m}$

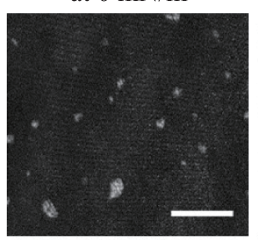

(c) before 1st ex. at $17 \mathrm{mN} / \mathrm{m}$ (d) before 2 nd com. at $0 \mathrm{mN} / \mathrm{m}$

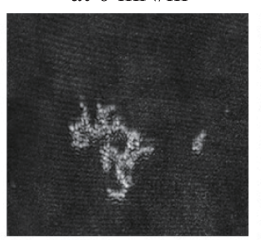

(e) before 2 nd ex at $17 \mathrm{mN} / \mathrm{m}$
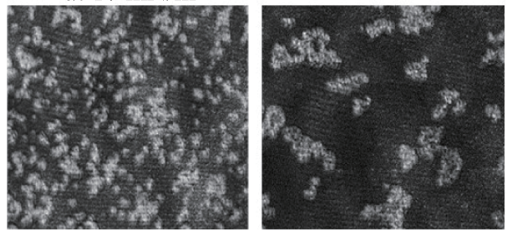

Fig. 5 (a) $\pi-A$ isotherms and (b - f) BAM images of MO/meibum $\Delta$ nonpolar-lipid (1:1) film in compression-expansion cycles (five times) at $20^{\circ} \mathrm{C}$. The images (b), (d), and (f) were taken at $0 \mathrm{mN} / \mathrm{m}\left(A \approx 73 \times 10^{5} \mathrm{~cm}^{2} / \mathrm{g}\right)$ just before starting the first and second compressions and after the fifth expansion, respectively. The images (c) and (e) were observed at 17 $\mathrm{mN} / \mathrm{m}$ just before starting the first and second expansions, respectively. A bar in (b) corresponds to $50 \mu \mathrm{m}$. 
(a) $\pi$ - $A$ isotherms

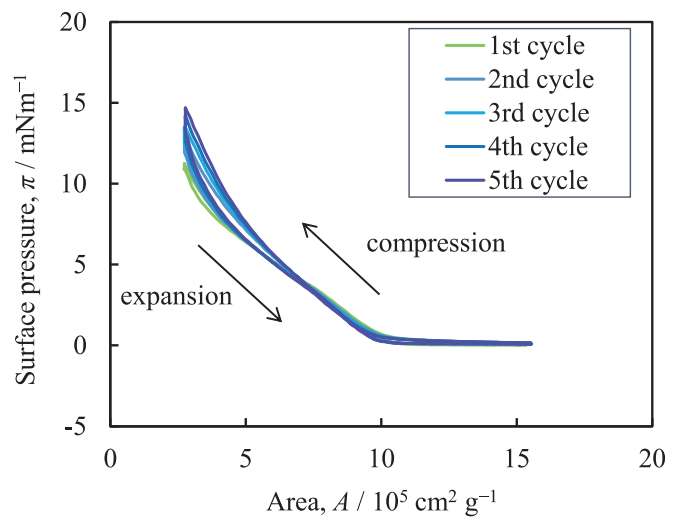

(b) before 1 st com. at $0 \mathrm{mN} / \mathrm{m}$

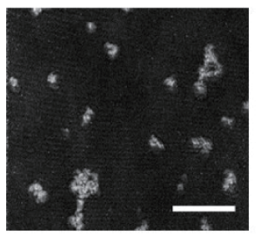

(c) before 1 st ex at $11 \mathrm{mN} / \mathrm{m}$

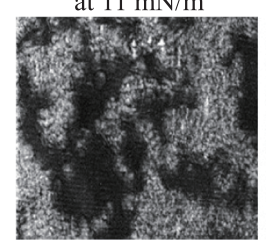

(d) before 2 nd com. at $0 \mathrm{mN} / \mathrm{m}$

(f) after 5 th ex.

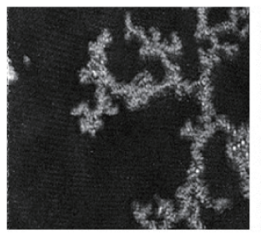

(e) before 2 nd ex at $13 \mathrm{mN} / \mathrm{m}$

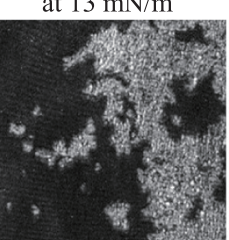

Fig. 6 (a) $\pi-A$ isotherms and (b-f) BAM images of MO/meibum $\Delta$ nonpolar-lipid (10:1) film in compression-expansion cycles (five times) at $20^{\circ} \mathrm{C}$. The images (b), (d), and (f) were taken at $0 \mathrm{mN} / \mathrm{m}\left(A \approx 15 \times 10^{5} \mathrm{~cm}^{2} / \mathrm{g}\right)$ just before starting the first and second compressions and after the fifth expansion, respectively. The images (c) and (e) were observed at 11 and $13 \mathrm{mN} / \mathrm{m}$ just before starting the first and second expansions, respectively. A bar in (b) corresponds to $50 \mu \mathrm{m}$.

served for the other films was not observed in the isotherms, although a kink was expected to appear below $15 \mathrm{mN} / \mathrm{m}$, according to the decreasing trend of surface pressure of the kink with increasing MO content(i.e., approximately $15.6,13.5$, and $12.5 \mathrm{mN} / \mathrm{m}$ for the films of meibum $\Delta$ nonpolar-lipid, MO/meibum $\Delta$ nonpolar-lipid (0.2:1), and $\mathrm{MO} /$ meibum $\Delta$ nonpolar-lipid (1:1), respectively). The BAM image (b)implied that some granular spots were already interconnected after spreading because of the high MO content. Then, the spots were gathered by the first compression (image (c)) and redispersed as interconnected structures upon expansion(image (d)). In the successive compression-expansion cycles, the interconnected structures were gathered and redispersed repeatedly (image (e) and (f)), similar to those observed in the MO/ meibum $\Delta$ nonpolar-lipid (1:1) film.

\section{Discussion}

Meibum $\Delta$ nonpolar-lipid, which is composed of polar lipids obtained from rabbit meibum through column chromatography, formed a spread monolayer at the air/water interface. The monolayer was in a homogeneous fluid phase, which was consistent with our previous insight as well as the commonly accepted expectation for the polarlipid monolayer regions between nonpolar lipid networks in meibum films ${ }^{16,18)}$. It was also confirmed in the present study that only the polar lipids did not form the condensed-phase networks, such as those observed in the meibum film. This observation suggested that the networks in the meibum film were primarily composed of nonpolar lipids. In contrast, the meibum $\Delta$ nonpolar-lipid monolayer reversibly formed aggregates at pressures above the surface pressure of the kink, upon compression, which could be related to the reversible formation of collapsed aggregates in the monolayer regions of the meibum film ${ }^{18)}$ and furthermore the stability of the TFLL on the eye against blinking. However, the cyclic compression and expansion for the meibum $\Delta$ nonpolar-lipid monolayer induced a shift of the $\pi-A$ isotherm to a smaller film area with the increase in cycle number, which was in contrast with the meibum film where the isotherms in the cycles were highly reversible ${ }^{18)}$. There are two possible reasons for the area shift in the cyclic isotherms of the meibum $\Delta$ nonpolar-lipid monolayer. One is the irreversible partial collapse of the monolayer, resulting in the formation of collapsed aggregates at the air/water interface, and the other is the dissolution of some film molecules into the subphase water due to compression. If the former is the case, the irreversibly collapsed aggregates would be too small to be observed by $\mathrm{BAM}$ at the air/water interface because no aggregates were observed in the film after the second and fifth cycle (Fig. 3 (d) and (f)).

The addition of $\mathrm{MO}$ to meibum $\Delta$ nonpolar-lipid changed the resultant film properties. The surface pressure of the kink in the isotherm decreased with the increase in MO fraction, up to the mixing ratio of MO:meibum $\Delta$ nonpolarlipid $=1: 1$, suggesting that $\mathrm{MO}$ and the meibum $\Delta$ nonpolarlipid molecules were mixed at certain ratios in the mixed films. However, the fraction of MO molecules in the monolayer phase was not very large, because the apparent shape of the isotherm was not very different in the MO/meibum$\Delta$ nonpolar-lipid (0.2:1) film in spite of the presence of granular spots of MO or MO-rich phase. Nevertheless, it was also confirmed that the quantitative relationship between MO and meibum $\Delta$ nonpolar-lipid influenced the degree of isotherm shift in the compression-expansion cycles. These observations suggest that the partial mixing along with the coexistence of granular spots and monolayer phase at ap- 

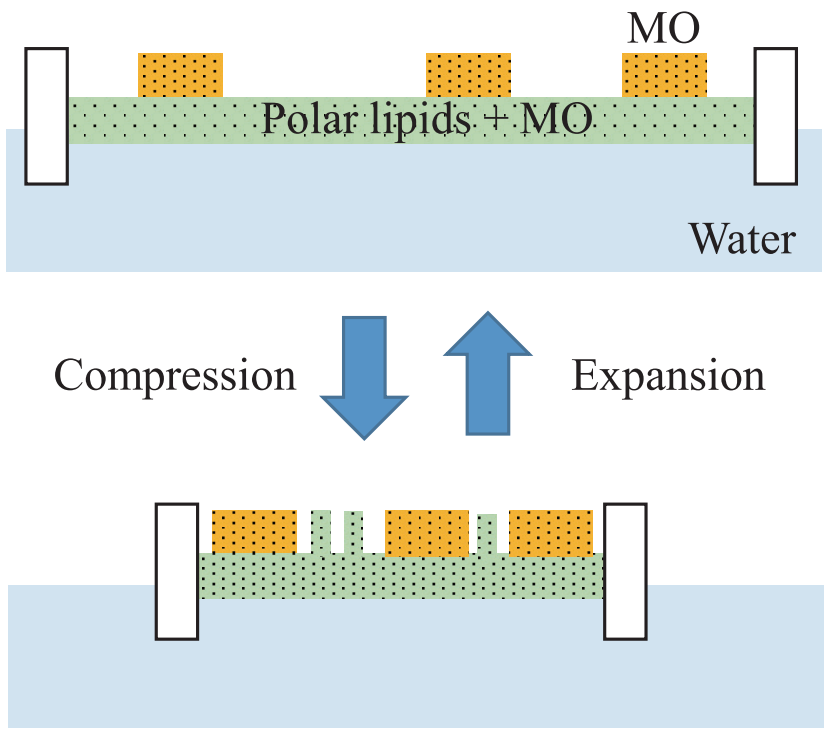

Fig. 7 Schematic illustration of the proposed structure of $\mathrm{MO} / \mathrm{meibum} \Delta$ nonplar-lipid film on the water surface. The MO/meibum $\Delta$ nonplar-lipid monolayer is composed of polar lipids and MO. The monolayer is partially collapsed upon compression but the collapsed aggregates returned to the monolayer upon expansion.

propriate mixing ratios can suppress the isotherm shift and thus recover the reversibility of repeated compression-expansion isotherms (Fig. 7).

\section{Conclusions}

This study analyzed the properties and morphology of the spread monolayer of meibum $\Delta$ nonpolar-lipid and the addition of MO to the monolayer system. The meibum$\Delta$ nonpolar-lipid formed a homogeneous fluid-phase monolayer - the area of which decreased with successive compression-expansion cycles, although the change in the film morphology was highly reversible throughout the cycles. However, this shift in the isotherm was suppressed by adding hydrophobic MO to the spreading solution at a mixing ratio of MO:meibum $\Delta$ nonpolar-lipid = 1:1 by weight. BAM observations of the $\mathrm{MO} /$ meibum $\Delta$ nonpolar-lipid mixed film implied the coexistence of granular spots of MO or MO-rich phase and the monolayer of meibum $\Delta$ nonpolarlipid rich phase. Such coexistent states, under appropriate quantitative relationships between MO and meibum$\Delta$ nonpolar-lipid, would be beneficial for suppressing the isotherm shift. Our results suggest that the addition of MO will be useful for regulating the film properties and structure.

\section{References}

1) Bron, A.J.; Tiffany, J.M.; Gouveia, S.M.; Yokoi, N.; Voon, L.W. Functional aspects of the tear film lipid layer. Exp. Eye Res. 78, 347-360 (2004).

2) Driver, P.J.; Lemp, M.A. Meibomian gland dysfunction. Surv. Ophthalmol. 40, 343-367(1996).

3) Lam, S.M.; Tong, L.; Duan, X.; Petznick, A.; Wenk, M.R.; Shui, G. Extensive characterization of human tear fluid collected using different techniques unravels the presence of novel lipid amphiphiles. J. Lipid Res. 55, 289-298 (2014).

4) McCulley, J.P.; Shine, W.E. A compositional based model for the tear film lipid layer. Trans. Am. Ophthal. Soc. 95, 79-93(1997).

5) Rosenfeld, L.; Cerretani, C.; Leiske, D.L.; Toney, M.F.; Radke, C.J.; Fuller, G.G. Structural and rheological properties of meibomian lipid. Invest. Ophth. Vis. Sci. 54, 2720-2732 (2013).

6) Rosenfeld, L.; Fuller, G.G. Consequences of interfacial viscoelasticity on thin film stability. Langmuir 28, 14238-14244(2012).

7) Patterson, M.; Vogel, H.J.; Prenner, E.J. The effect of repeated lateral compression and expansions mimicking blinking on selected tear film polar lipid monofilms. Biochim. Biophys. Acta 1859, 319-330 (2017).

8) Liu, X; Riess, G.J; Krafft, P.M. Self-Organization of semifluorinated alkanes and related compounds at interfaces: thin films, surface domains and two-dimensional spherulites. Bull. Chem. Soc. Jpn. 91, 846-857 (2018).

9) Ariga, K.; Mori, T.; Li, J. Langmuir Nanoarchitectonics from basic to frontier. Langmuir 35, 3585-3599 (2019).

10) Nakahara, H. Fluidizing and solidifying effects of perfluorooctylated fatty alcohols on pulmonary surfactant monolayers. J. Oleo Sci. 65, 99-109 (2016).

11) Nakahara, H.; Shibata, O. Miscibility of semifluorinated pentadecanol with DPPC at the air-water interface. Acc. Mater. Surf. Res. 3, 199-208(2018).

12) Sachan, A.K.; Zasadzinski, J.A. Interfacial curvature effects on the monolayer morphology and dynamics of a clinical lung surfactant. Proc. Natl. Acad. Sci. USA 115, E134-E143(2018).

13) Arciniega, J.C.; Nadji, E.J.; Butovich, I.A. Effects of free fatty acids on meibomian lipid films. Exp. Eye Res. 93, 452-459(2011).

14) Schuett, B.S.; Millar, T.J. An investigation of the likely role of (O-acyl) $\omega$-hydroxy fatty acids in meibomian lipid films using (O-oleyl) $\omega$-hydroxy palmitic acid as a model. Exp. Eye Res. 115, 57-64(2013).

15) Arciniega, J.C.; Uchiyama, E.; Butovich, I.A. Disruption and destabilization of meibomian lipid films caused by increasing amounts of ceramides and cholesterol. Invest. Ophth. Vis. Sci. 54, 1352-1360 
(2013).

16) Millar, T.J. A mechanism to explain the behavior of spread films of meibomian lipids. Cur. Eye Res. 38, 220-223 (2013).

17) Georgiev, G.A.; Borchman, D.; Eftimov, P.; Yokoi, N. Lipid saturation and the rheology of human tear lipids. Int. J. Mol. Sci. 20, E3431 (2019).

18) Yoshida, M.; Yamaguchi, M.; Sato, A.; Tabuchi, N.; Kon, R.; Iimura, K. Role of endogenous ingredients in meibum and film structures on stability of tear film lipid layer against lateral compression. Langmuir 35, 8445-8451 (2019).

19) Pérez-Gil, J.; Keough, K.M. Interfacial properties of surfactant proteins. Biochim. Biophys. Acta 1408, 203-217 (1998).

20) Phan, M.D.; Lee, J.; Shin, K. Collapsed states of Langmuir monolayers. J. Oleo Sci. 65, 385-397(2016). 